\title{
Static quark-antiquark pair free energy and screening masses: continuum results at the QCD physical point
}

\section{Szabolcs Borsányi}

University of Wuppertal

\section{Zoltán Fodor}

University of Wuppertal

\section{Sándor D. Katz}

Eötvös University and MTA-ELTE Lendület Lattice Gauge Theory Research Group

\author{
Attila Pásztor* \\ University of Wuppertal \\ E-mail: apasztor@bodri.elte.hu
}

\section{Kálmán K. Szabó}

University of Wuppertal and Jülich Supercomputing Center

\section{Csaba Török}

Eötvös University and MTA-ELTE Lendület Lattice Gauge Theory Research Group

\begin{abstract}
We study the correlators of Polyakov loops, and the corresponding gauge invariant free energy of a static quark-antiquark pair in 2+1 flavor QCD at finite temperature. Our simulations were carried out on $N_{t}=6,8,10,12,16$ lattices using a Symanzik improved gauge action and a stout improved staggered action with physical quark masses. The free energies calculated from the Polyakov loop correlators are extrapolated to the continuum limit. For the free energies we use a two step renormalization procedure that only uses data at finite temperature. We also measure correlators with definite Euclidean time reversal and charge conjugation symmetry to extract two different screening masses, one in the magnetic, and one in the electric sector, to distinguish two different correlation lengths in the full Polyakov loop correlator. This conference contribution is based on the paper: JHEP 1504 (2015) 138
\end{abstract}

The 33rd International Symposium on Lattice Field Theory

14 -18 July 2015

Kobe International Conference Center, Kobe, Japan

${ }^{*}$ Speaker. 


\section{Introduction}

At high temperatures strongly interacting matter undergoes a transition where colorless hadrons turn into a phase dominated by colored quarks and gluons, the quark gluon plasma (QGP). Deconfinement properties of the transition can be studied by infinitely heavy, static test charges. Here, we calculate the gauge invariant static quark-antiquark pair free energy.

The free energy of a static quark-antiquark pair as a function of their distance at various temperatures is determined by the Polyakov loop correlator [1], which gives the gauge invariant $\bar{Q} Q$ free energy ${ }^{1}$ as:

$$
F_{\bar{Q} Q}(r)=-T \ln C(r, T)=-T \ln \left\langle\sum_{\mathbf{x}} \operatorname{Tr} L(\mathbf{x}) \operatorname{Tr} L^{+}(\mathbf{x}+\mathbf{r})\right\rangle .
$$

In the above formula, $\mathbf{x}$ runs over all the lattice spatial sites, and the Polyakov loop, $L(\mathbf{x})$, is defined as the product of temporal link variables ${ }^{2} U_{4}\left(\mathbf{x}, x_{4}\right) \in S U(3)$ :

$$
L(\mathbf{x})=\prod_{x_{4}=0}^{N_{t}-1} U_{4}\left(\mathbf{x}, x_{4}\right),
$$

A related problem is distinguishing correlation lengths in the correlator of Polyakov loops, which give inverse screening masses in the plasma. In the full Polyakov loop correlator the electric and magnetic sectors both contribute. In order to investigate the effect of electric and magnetic gluons separately, one can use the symmetry of Euclidean time reflection [2], that we will call $\mathscr{R}$. The crucial property of magnetic versus electric gluon fields $A_{4}$ and $A_{i}$ is that under this symmetry, one is intrinsically odd, while the other is even:

$$
A_{4}(\tau, \mathbf{x}) \stackrel{\mathscr{R}}{\rightarrow}-A_{4}(-\tau, \mathbf{x}), \quad \mathrm{A}_{\mathrm{i}}(\tau, \mathbf{x}) \stackrel{\mathscr{R}}{\rightarrow} \mathrm{A}_{\mathrm{i}}(-\tau, \mathbf{x})
$$

Under this symmetry the Polyakov loop transforms as $L \stackrel{\mathscr{R}}{\rightarrow} L^{\dagger}$. One can easily define correlators that are even or odd under this symmetry, and thus receive contributions only from the magnetic or electric sector, respectively [2,3]:

$$
L_{M} \equiv\left(L+L^{\dagger}\right) / 2 \quad L_{E} \equiv\left(L-L^{\dagger}\right) / 2 .
$$

We can further decompose the Polyakov loop into $\mathscr{C}$ even and odd states, using $A_{4} \stackrel{\mathscr{C}}{\rightarrow} A_{4}^{*}$ and $L \stackrel{\mathscr{C}}{\rightarrow} L^{*}$ as:

$$
L_{M \pm}=\left(L_{M} \pm L_{M}^{*}\right) / 2 \quad L_{E \pm}=\left(L_{E} \pm L_{E}^{*}\right) / 2 .
$$

Next, we note that $\operatorname{Tr} L_{E+}=0=\operatorname{Tr} L_{M-}$, so the decomposition of the Polyakov loop correlator to definite $\mathscr{R}$ and $\mathscr{C}$ symmetric operators contains two parts ${ }^{3}$. We define the magnetic correlation

\footnotetext{
${ }^{1}$ More precisely, the excess free energy that we get when inserting two static test charges in the medium.

${ }^{2}$ In the literature, a factor of $\frac{1}{N_{c}}$ is often included in the definition. Including this factor leads to a term in the static quark free energy that is linear in temperature.

${ }^{3}$ Note that the Polyakov loop correlator does not overlap with the $\mathscr{R}(\mathscr{C})=+(-)$ and $\mathscr{R}(\mathscr{C})=-(+)$ sectors. To access these sectors, other operators are needed.
} 
function as:

$$
C_{M+}(r, T) \equiv\left\langle\sum_{\mathbf{x}} \operatorname{Tr} L_{M+}(\mathbf{x}) \operatorname{Tr} L_{M+}(\mathbf{x}+\mathbf{r})\right\rangle-\left|\left\langle\sum_{\mathbf{x}} \operatorname{Tr} L(\mathbf{x})\right\rangle\right|^{2},
$$

and the electric correlator as ${ }^{4}$ :

$$
C_{E-}(r, T) \equiv-\left\langle\sum_{\mathbf{x}} \operatorname{Tr} L_{E-}(\mathbf{x}) \operatorname{Tr} L_{E-}(\mathbf{x}+\mathbf{r})\right\rangle .
$$

Then, from the exponential decay of these correlators, we can define the magnetic and electric screening masses. Note that with our definition $\operatorname{Tr} L_{M+}=\operatorname{Re} \operatorname{Tr} L$ and $\operatorname{Tr} L_{E-}=i \operatorname{Im} \operatorname{Tr} L$, and:

$$
C(r, T)-C(r \rightarrow \infty, T)=C_{M+}(r, T)+C_{E-}(r, T),
$$

from which it trivially follows that if the magnetic mass screening mass is lower than the electric mass, we will have $C(r, T)-C(r \rightarrow \infty, T)$ asymptotic to $C_{M+}(r, T)$ as $r \rightarrow \infty$, or equivalently, the highest correlation length in $C$ equal to that of $C_{M+}$. We will determine the correlation lengths by fitting a Yukawa ansatz to these correlators.

\section{Simulation details}

The simulations were performed by using the tree level Symanzik improved gauge, and stoutimproved staggered fermion action, that was used in [4]. We worked with physical quark masses, and fixed them by reproducing the physical ratios $m_{\pi} / f_{K}$ and $m_{K} / f_{K}$.

Compared to our previous investigations of Polyakov loop correlators, reported in the conference proceedings [5], here we used finer lattices, namely we carried out simulations on $N_{t}=12$ and 16 lattices as well as on $N_{t}=6,8,10$ lattices. Our results were obtained in the temperature range $150 \mathrm{MeV} \leq \mathrm{T} \leq 450 \mathrm{MeV}$. We use the same configurations as in [6] and [7].

\section{The gauge invariant free energy}

\subsection{Renormalization}

We use a renormalization procedure based entirely on our $T>0$ data, similarly to Refs. [8] and [9]. The data contains a temperature independent divergent part from the ground state energy. The difference between the value of free energies at different temperatures is free of divergences. Accordingly, we define the renormalized free energy as:

$$
F_{\bar{Q} Q}^{r e n}\left(r, \beta, T ; T_{0}\right)=F_{\bar{Q} Q}(r, \beta, T)-F_{\bar{Q} Q}\left(r \rightarrow \infty, \beta, T_{0}\right),
$$

with a fixed $T_{0}$. This renormalization prescription corresponds to the choice that the free energy at large distances goes to zero at $T_{0}$. We choose $T_{0}=200 \mathrm{MeV}$. Our renormalization procedure is implemented in two steps.

\footnotetext{
${ }^{4}$ Here our definition differs from that used in [3] in a sign.
} 


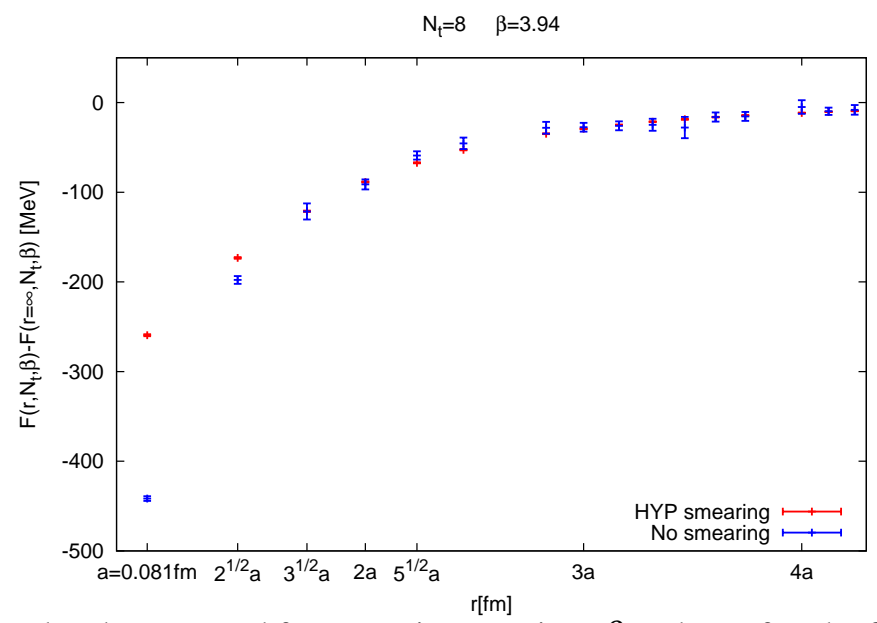

Figure 1: The smeared and unsmeared free energies at a given $\beta$ and $N_{t}$, after the first step of the renormalization procedure.

In the first step we renormalize the single static quark free energy which satisfies:

$$
2 F_{Q}(\beta, T)=F_{\bar{Q} Q}(r \rightarrow \infty, \beta, T)=-T \log |\langle\operatorname{Tr} L\rangle|^{2} .
$$

We define its renormalized counterpart as:

$$
F_{Q}^{r e n}\left(\beta, T ; T_{0}\right)=F_{Q}(\beta, T)-F_{Q}\left(\beta, T_{0}\right) .
$$

In the second step the full renormalized $\bar{Q} Q$ free energy can be written as:

$$
F_{\bar{Q} Q}^{r e n}\left(r, \beta, T ; T_{0}\right)=\tilde{F}_{\bar{Q} Q}(r, \beta, T)+2 F_{Q}^{r e n}\left(\beta, T ; T_{0}\right),
$$

where

$$
\tilde{F}_{\bar{Q} Q}(r, \beta, T)=F_{\bar{Q} Q}(r, \beta, T)-F_{\bar{Q} Q}(r \rightarrow \infty, \beta, T)=F_{\bar{Q} Q}(r, \beta, T)-2 F_{Q}(\beta, T) .
$$

Note, that this second step of the renormalization procedure is completely straightfoward to implement, at each simulation point in $N_{t}$ and $\beta$ we just subtract the asymptotic value of the correlator. The main advatage of this 2 step procedure is that it allows us to extend the temperature range we can do a continuum limit in without performing $T=0$ simulations at lots of different $\beta$ values. For more details see [11].

\subsection{Smearing as variance reduction}

The Polyakov loop correlator behaves similarly to baryon correlators in imaginary time: at large values of $r$ we can get negative values of $C$ at some configurations ${ }^{5}$. For this reason, it is highly desirable to use gauge field smearing which makes for a much better behavior at large $r$, at the expense of unphysical behavior at small $r$. For this reason, we measured the correlators both without and with HYP smearing. We expect that outside the smearing range (i.e. $r \geq 2 a$ ) the two correlators coincide. This is supported by Figure 1. Therefore we use the smeared correlators for $r \geq 2 a$ and the unsmeared ones for $r<2 a$.

\footnotetext{
${ }^{5}$ Of course, the ensemble average should in principle be positive definite.
} 


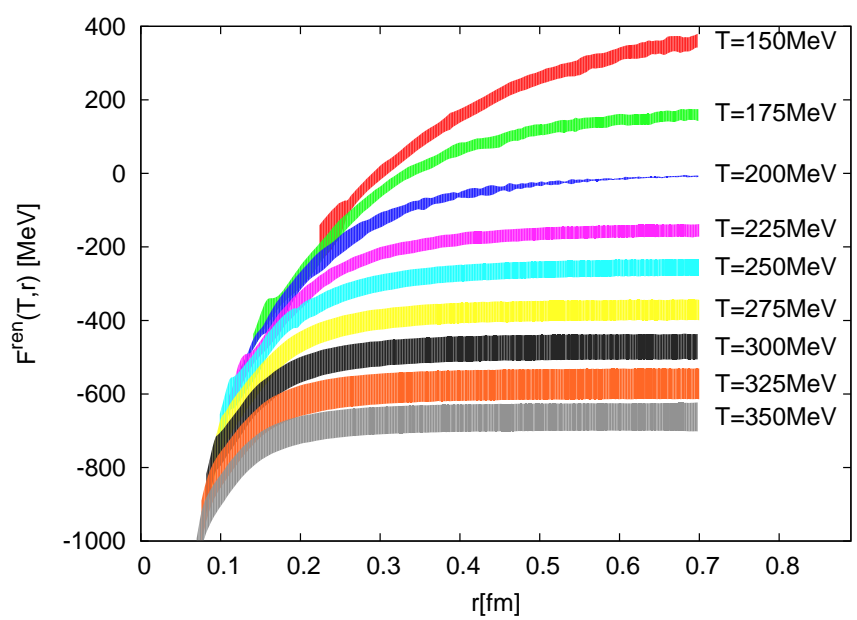

Figure 2: Continuum values of the static $\bar{Q} Q$ free energy at different temperatures.

\subsection{Results}

The continuum extrapolations were done with $N_{t}=8,10,12$ and when available $N_{t}=16$ lattices. For details on the systematic error estimation see [11]. The final results are in Figure 2. Note that the curves seem to tend to the same curve as $r \rightarrow 0$, corresponding to the expectation that UV physics is temperature independent. Also note that the error bars get smaller as we approach $T_{0}=200 \mathrm{MeV}$, which was chosen as a renormalization point. This is a natural consequence of the implementation of our renormalization prescription. It is also the reason why the correlator tends to zero at that point. A different renormalization would correspond to a constant shift in this graph.

We note, that on this conference an other determination of the same free energy was reported [12] using the HISQ action, and there is a slight difference in the asymptotic value (or the single quark free energy), at the higher temperature values of $T>300 \mathrm{MeV}$. It amounts to approximately $1-1.5 \sigma$. In the lower temperature range, where published continuum data on the Polyakov loop is also available, we have an agreement, see e.g. [13].

\section{The screening masses}

We continue with the discussion of the electric and magnetic screening masses obtained from the correlators (1.6) and (1.7). For this analysis we only use lattices above the (pseudo)critical temperature, since that is the physically interesting range for screening. Next, we mention that for this analysis, we only use the data with HYP smearing, since we are especially interested in the large $r$ behavior. To chose the correct fit interval for the Yukawa ansatz, we use the KolmogorovSmirnov test to check whether the $\chi^{2}$-s are properly distributed. Note that the determination of the screening masses does not need additional renormalization. We fit linear functions to all screening masses at all values of $N_{t}$, and use these to do a continuum extrapolation from the $N_{t}=8,10,12$ lattices. The final results, along with some comparisons with results from the literature are in Fig. 3. For details on the fitting procedure and the systematic error estimation see [11]. 

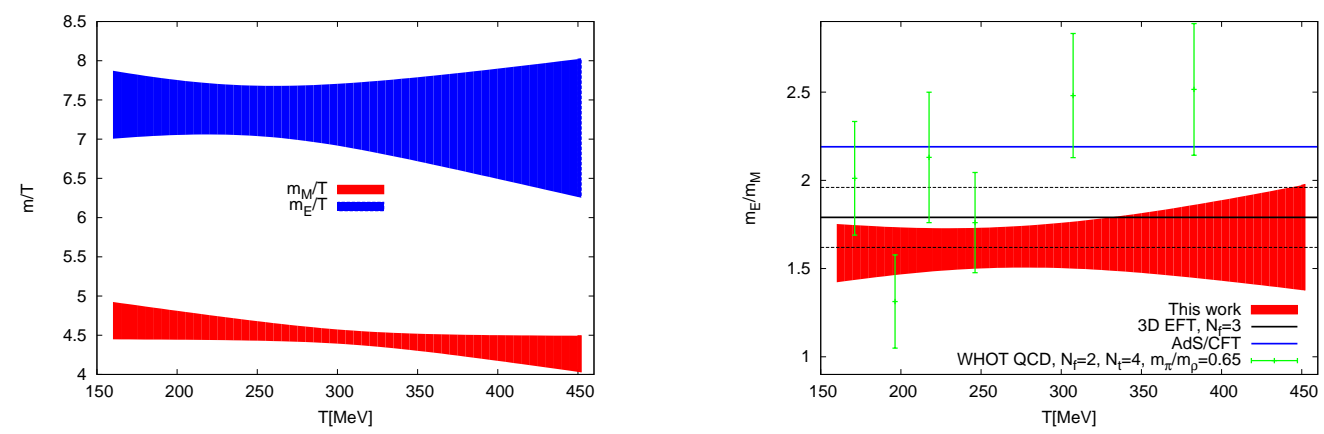

Figure 3: The continuum extrapolations of the screening masses and the ratio of the screening masses. For the ratio $m_{E} / m_{M}$ we also included different estimates from the literature: Lattice results from Ref. [3], dimensionally reduced 3D effective field theory results at $T=2 T_{c}$ from Ref. [14], and results from $\mathscr{N}=4$ SYM plasma with AdS/CFT from Ref. [15].

Figure 3: The continuum extrapolations of the screening masses and the ratio of the screening masses. For the ratio $m_{E} / m_{M}$ we

\subsection{Comparison with the literature}

We finish this section by comparing our results to those from earlier approximations in the literature. For comparison let us use our results at $T=300 \mathrm{MeV} \approx 2 \mathrm{~T}_{\mathrm{c}}$. Here we have:

- This work: $2+1$ flavour lattice QCD at the physical point after continuum extrapolation:

$$
m_{E} / T=7.31(25) \quad m_{M} / T=4.48(9) \quad m_{E} / m_{M}=1.63(8)
$$

- Ref. [3]: 2 flavour lattice QCD with Wilson quarks, a somewhat heavy pion $m_{\pi} / m_{\rho}=0.65$, no continuum extrapolation

$$
m_{E} / T=13.0(11) \quad m_{M} / T=5.8(2) \quad m_{E} / m_{M}=2.3(3)
$$

- From Table 1 of Ref. [15]: $\mathscr{N}=4 \mathrm{SYM}$, large $N_{c}$ limit, AdS/CFT

$$
m_{E} / T=16.05 m_{M} / T=7.34 m_{E} / m_{M}=2.19
$$

- From Figure 3 of Ref. [14]: dimensionally reduced 3D effective theory, $N_{f}=2$ massless quarks

$$
m_{E} / T=7.0(3) \quad m_{M} / T=3.9(2) \quad m_{E} / m_{M}=1.79(17)
$$

- From Figure 3 of Ref. [14]: dimensionally reduced 3D effective theory, $N_{f}=3$ massless quarks

$$
m_{E} / T=7.9(4) \quad m_{M} / T=4.5(2) \quad m_{E} / m_{M}=1.76(17)
$$

We note, that our results are closest to the results from dimensionally reduced effective field theory. 


\section{Summary}

In this paper we have determined the renormalized static quark-antiquark free energies in the continuum limit. We introduced a two step renormalization procedure using only the finite temperature results. The low radius part of the free energies tended to the same curve, corresponding to the expectation that at small distances, the physics is temperature independent. We also calculated the magnetic and electric screening masses, from the real and imaginary parts of the Polyakov loop respectively. As expected, both of these masses approximately scale with the temperature as $m \propto T$, with $m_{M}<m_{E}$, therefore, magnetic contributions dominating at high distances. The values we got for the screening masses are close to the values from dimensionally reduced effective field theory.

\section{Acknowledgment}

Computations were carried out on GPU clusters at the Universities of Wuppertal and Budapest as well as on supercomputers in Forschungszentrum Juelich. This work was supported by the DFG Grant SFB/TRR 55, ERC no. 208740. and the Lendulet program of HAS (LP2012-44/2012).

\section{References}

[1] L. D. McLerran and B. Svetitsky, Phys. Rev. D 24, 450 (1981).

[2] P. B. Arnold and L. G. Yaffe, Phys. Rev. D 52, 7208 (1995) [hep-ph/9508280].

[3] Y. Maezawa et al. Phys. Rev. D 81, 091501 (2010) [arXiv:1003.1361 [hep-lat]].

[4] Y. Aoki, Z. Fodor, S. D. Katz and K. K. Szabo, JHEP 0601, 089 (2006) [hep-lat/0510084].

[5] Z. Fodor, A. Jakovac, S. D. Katz and K. K. Szabo, PoS LAT 2007, 196 (2007) [arXiv:0710.4119 [hep-lat]].

[6] S. Borsanyi et al. [Wuppertal-Budapest Collaboration], JHEP 1009, 073 (2010) [arXiv:1005.3508 [hep-lat]].

[7] S. Borsanyi, Z. Fodor, C. Hoelbling, S. D. Katz, S. Krieg and K. K. Szabo, Phys. Lett. B 730, 99 (2014) [arXiv:1309.5258 [hep-lat]].

[8] S. Gupta, K. Huebner and O. Kaczmarek, Phys. Rev. D 77, 034503 (2008) [arXiv:0711.2251 [hep-lat]].

[9] S. Borsanyi et al., JHEP 1208, 126 (2012) [arXiv:1205.0440 [hep-lat]].

[10] S. Borsanyi, Z. Fodor, S. D. Katz, A. Pasztor, K. K. Szabo and C. Torok, JHEP 1504, 138 (2015) [arXiv:1501.02173 [hep-lat]].

[11] S. Borsanyi, Z. Fodor, S. D. Katz, A. Pasztor, K. K. Szabo and C. Torok, JHEP 1504, 138 (2015) [arXiv:1501.02173 [hep-lat]].

[12] J. Weber, A. Bazavov, N. Brambilla. M.Berwein, P. Petrezcky and A. Vairo This proceedings

[13] A. Bazavov and P. Petreczky, Phys. Rev. D 87, no. 9, 094505 (2013) [arXiv:1301.3943 [hep-lat]].

[14] A. Hart, M. Laine and O. Philipsen, Nucl. Phys. B 586, 443 (2000) [hep-ph/0004060].

[15] D. Bak, A. Karch and L. G. Yaffe, JHEP 0708, 049 (2007) [arXiv:0705.0994 [hep-th]]. 